\title{
Structure based virtual screening, molecular docking and molecular dynamics simulation of drug like small molecules against cell cycle regulator enzyme FtsZ of Mycobacterium tuberculosis
}

Partha Sarathi Mohanty ( $\nabla$ m.sarathipartha@gmail.com )

National JALMA Institute for Leprosy and Other Mycobacterial Diseases

Roopendra Singh

National JALMA Institute for Leprosy and Other Mycobacterial Diseases

Nisha Pawar

Dr BR Ambedkar University

Chayanika Gogoi

Central University of Punjab

Sandeep Sharma

National JALMA Institute for Leprosy and Other Mycobacterial Diseases

Harpreet Singh Pawar

National JALMA Institute for Leprosy and Other Mycobacterial Diseases

Keshar Kunja Mohanty

National JALMA Institute for Leprosy and Other Mycobacterial Diseases

Research Article

Keywords: Mycobacterium, Tuberculosis, FtsZ, Docking

Posted Date: January 9th, 2021

DOI: https://doi.org/10.21203/rs.3.rs-142773/v1

License: (c) (i) This work is licensed under a Creative Commons Attribution 4.0 International License.

Read Full License 


\section{Structure based virtual screening, molecular docking and molecular dynamics}

simulation of drug like small molecules against cell cycle regulator enzyme FtsZ of

\section{Mycobacterium tuberculosis}

Partha Sarathi Mohanty

Scientist-D

Department of Epidemiology

National JALMA Institute for Leprosy and Other Mycobacterial Diseases

M. Miyazaki Marg, Tajganj, Agra, India

mohanty.ps@icmr.gov.in

Roopendra Kumar

Research Assistant

Department of Epidemiology

National JALMA Institute for Leprosy and Other Mycobacterial Diseases

M. Miyazaki Marg, Tajganj, Agra, India

singhrrp1998@gmail.com

Nisha Pawar

Master of Biotechnology student

School of Life Sciences, Dr. BR Amedkar University

Khandari, Agra, India

nishapawar700@gmail.com

Chayanika Gogoi

Master of Biochemistry student

Department of Biochemistry

Central University of Punjab

Bhatinda, India

chayanika354@gmail.com

Sandeep Sharma

Scientist-B

Department of Epidemiology

National JALMA Institute for Leprosy and Other Mycobacterial Diseases

M. Miyazaki Marg, Tajganj, Agra, India

drsandeepsharma82@yahoo.in

Harpreet Singh Pawar

Scientist-B

Department of Clinics

National JALMA Institute for Leprosy and Other Mycobacterial Diseases

M. Miyazaki Marg, Tajganj, Agra, India

harpreet.pawar@icmr.gov.in

Keshar Kunja Mohanty

Scientist-F

Department of Immunology

National JALMA Institute for Leprosy and Other Mycobacterial Diseases

M. Miyazaki Marg, Tajganj, Agra, India

keshar63@yahoo.com 


\section{Abstract}

Tuberculosis is a major public health problem in several developing and low income countries. It warranted 1.2 million deaths around the world in 2019. An active Tb patient can spread a large number of bacilli to the environment by coughing, spitting, speaking and sneezing which in turn help in development of new Tb cases. Further development of drug resistant strains of Mycobacterium tuberculosis to most powerful first line drugs viz., rifampicin and isoniazid put a hurdle in Tb elimination programme. Thus it is essential to explore new drug molecules to fight against $\mathrm{Tb}$ morbidity and mortality. In the present study an attempt has been made to screen new small drug like molecules using structure based molecular screening. 3D structure of cell cycle regulator enzyme FtsZ (PDB ID: 1RLU) was retrieved from the Protein Data Bank website and used it for structure-based virtual screening of drug like small molecules from PubChem database. The screened drugs were re-docked with FtsZ of M. tuberculosis to find out their affinity and antagonistic effect against M. tuberculosis. Molecular dynamics simulation studies were carried out for each of the docked complex to find out the stability and flexibility of the docked conformations. In silico ADME study was carried out to find out the drug likeliness of the selected molecules. A total of 1501 drug like small molecules were recovered through virtual screening of which three molecules, viz., Lig-24284406, Lig49671233 and Lig-24791139 were taken for the docking study. It was observed that the selected drug molecules had a strong affinity towards the FtsZ. This was evident from the binding energy of the drug molecules towards FtsZ (-7.23, -9.67 and -5.51). The strong stability and flexibility of the docked structures were also evident from RMSD values i.e, $<2 \AA$ and RMSF value i.e, > $1 \AA$ for all the docking complexes. The present study suggested that three small drug like molecules could be taken for further clinical studies to find their efficacy against tuberculosis.

\section{Introduction}

Tuberculosis (Tb) is an infectious disease caused by Mycobacterium tuberculosis an acid fast bacterium of family Mycobacteriaceae. Despite of effective multi drug therapy $\mathrm{Tb}$ remains a major public health problem worldwide. Mainly the disease affects lungs and known as

pulmonary $\mathrm{Tb}$ but it can affect other parts of the body like bone, brain, skin, intestine etc. and is known as extra-pulmonary Tb. According to global tuberculosis report 2019 an estimated 10 million people were suffered from tuberculosis and an estimated 1.2 million $\mathrm{Tb}$ deaths were reported among HIV negative patients in 2018 (WHO 2020). Two third of the total TB cases were reported from eight countries viz., India (27\%), China (9\%), Indonesia (8\%), Philippines (6\%), Pakistan (6\%), Nigeria (4\%), Bangladesh (4\%) and South Africa (3\%) (WHO 2020). Tb 
is spread from an active $\mathrm{Tb}$ patient to a healthy person through air. A large number of bacilli were spread in the air by coughing, spitting, speaking and sneezing of an active $\mathrm{Tb}$ patient. People with latent $\mathrm{Tb}$ do not spread $\mathrm{Tb}$. Treatment of $\mathrm{Tb}$ requires a long time period with multiple antibiotics. Although $\mathrm{Tb}$ is curable disease but development and spread of drug resistance Tb viz., MDR (Multi Drug Resistant) and XDR (Extensive Drug Resistant) strains are major hurdle to achieve the goal of Tb elimination (Fox et al. 2017). Multi drug therapy for $\mathrm{Tb}$ therapy implemented to avoid complications, increase the cure rate, decrease the mortality rate, preventing recurrences and also to restrict the dissemination of drug resistant strains of $M$. tuberculosis (AlMatar et al. 2017). The development of resistance to most powerful Tb drug viz., rifampicin and isoniazid and horizontal transfer of MDR strains in the community (Anderson et al. 2014; Seung et al. 2015) put an obstacle in Tb elimination programme. Thus new antibiotics having pharmacological action against $M$. tuberculosis should be screened to treat both drug susceptible and resistant strains of $M$. tuberculosis to achieve the goal of elimination. Thus in this study FtsZ, a cell cycle regulator enzyme has been used as drug target to screen small molecule using structure based drug screening.

FtsZ is an essential gene for in vitro growth of M. tuberculosis (Griffin et al. 2011; DeJesus et al. 2017). Fts $Z$ is involved in $\mathrm{Z}$ ring formation and the initiation of cell division (Bi, and Lutkenhaus 1991). FtsZ possesses many unique biochemical features, which include slow polymerisation kinetics, presence of charged amino acids in the C-terminal domain that interacts with a variety of other cell division proteins (Gupta et al. 2016). FtsZ is the most abundant cell division protein and plays an essential role in bacteria cytokinesis. The initiation of FtsZ polymerization requires GTP. The polymerization takes place in inner membrane at middle of the dividing cell and forwards bi directionally to form a highly dynamic helical structure known as the Z-ring that encircles the cell. Following recruitment of the other cell division proteins, Z-ring contracts to form septum (Huang et al. 2007).

Looking to the essential function of FtsZ protein in cell cycle regulation, an attempt has been made to screen small lead like molecules from PubChem small molecule database using 3D structure of FtsZ protein of M. tuberculosis and selected molecules were docked into the FtsZ and validated through molecular dynamics simulation analysis. The finding of this study might be able to provide a new paradigm for alternative regimen against MDR and XDR tuberculosis.

\section{Material and Methods:}

\subsection{Retrieval of Fts $Z$ and domain analysis}

The holoenzyme of FtsZ was retrieved from the protein data bank (PDB) under the accession number 1RLU (Leung et al. 2004). The crystal structure of FtsZ consisted two identical 
subunits, A and B. The enzyme is composed of $\alpha \beta$-tubulin dimer (Nogales et al. 1999) and microtubules (Li et al. 2007). Structure editing was done using Discovery Studio Visualizer. All the chains, hetero atoms and water were removed except chain 'A' containing the catalytic centre of the enzyme. The cleaned structure of FtsZ was saved in .pdb format for further processing. The protein family, superfamily and arrangement of domains were elucidated using InterProScan (Zdobnov and Apweiler 2001). Conserved domains of the FtsZ were investigated using the databases such as Pfam (Finn et al. 2014) and CDD (MarchlerBauer et al. 2009).

\subsection{Binding site selection}

The ligand binding sites were selected on the basis of binding of binding of GTP-gamma-S to original crystal structure (1RLU). These amino acid binding sites are Gly-17, Gly-18, Gly-19, Asn-22, Thr-42, Ala-52, Asp-53, Lys-55, Gly-67, Ala-68, Gly-69, Ala-70, Gly-101, Glu-102, Gly-104, Gly-105, Thr-106, Gly-107, Pro-132, Glu-136, Arg-140, Phe-180, Ala-183, Asp-184 and Leu-187.

\subsection{Preparation of protein, ligand, docking and visualization}

3D conformers of ligands having lowest binding energy in the screening process were downloaded from PubChem using PubChem compound CID as sdf format. In this study three small molecules viz., N,N-diethyl-6-methyl-2-oxo-1,3-dihydrobenzimidazole-5-sulfonamide (CID-24284406), N-[2-[3-(4-fluorophenyl)-6-oxopyridazin-1-yl]ethyl]-2-(4-methyl-6oxopyrimid in-1-yl)acetamide (CID-49671233) and [4-(hydroxymethyl)-4-[[3(trifluoromethyl)phenyl]methyl]piperidin-1-yl]-(5-methyl-1H-pyrazol-3-yl)methanone (CID24791139) were taken for further molecular docking and molecular dynamics simulation analysis. Further the compounds were described by their compound ID throughout the text. Then the sdf file was converted to .pdb using PyMol v-2.2.3 (DeLano et al. 2002). Enzyme (FtsZ) and ligands (24284406, 49671233 and 24791139) were prepared for docking using AutoDock MGLTtools v1.5.6 (Morris et al. 2009). The polar hydrogens were added to the protein using 'hydrogens' in 'edit option' and charges were added using 'charges' in 'edit option'. Then the enzyme was saved in pdbqt format. For flexible docking the catalytic binding sites in the FtsZ was selected and the grid box was modified accordingly. The dimensions of the grid box were written as grid.txt file. Likewise gasteiger charges and merging of non polar hydrogens were done using 'select molecule for AutoDock4' option in the ligand panel. Then the ligand was saved in pdbqt format. The flexible docking was done using AutoDock Vina v2.0 software package (Trott and Olson 2010).

\subsection{Molecular dynamics (MD) simulation of rpoB-resistomycin complex}


Molecular dynamics (MD) simulation is a principal tool for proofing the stability of proteinligand complex. MD simulation provides the detailed information on the fluctuations and conformational changes of proteins-ligand complex towards its stable conformation. Thus in the present study MD simulation was applied to understand the stability of FtsZ-ligand binding complexes. Files required for MD simulations were prepared using Visual Molecular Dynamics (VMD) v 1.9.3. Initially co-ordinates of FtsZ and ligands were written in .pdb format using save coordinates option of the VMD (Humphrey et al. 1996). The FtsZ was parameterized using automatic psf builder. For parameterization the topology parameter “top_all27_prot_lipid_na.inp was used". For parameterization of ligands ligand reader and modeller of CHARMM force filed was used (Jo et al. 2008) at Charmm Gui web site (http://www.charmm-gui.org/?doc=input). Then individual psf and pdb files of FtsZ and ligands were generated using TCL script of the VMD. The complexes were then solvated using TIP3P water box with $3.0 \AA$ of water in each direction of the coordinate structure. Integrator parameters were included as $2 \mathrm{fs} / \mathrm{step}$ for all rigid bonds and nonbonded frequencies at $2.5 \AA$ and electrostatic evaluations for $3 \AA$ with ten steps for each cycle. Periodic boundary conditions and PME were adjusted using maxmin TCL script. Water constraints were eliminated by minimizing preliminary protein energy via 1000 steps of the Powel algorithm at a constant temperature of 310 K. Simulations were run for 200,000 runs for $2 \mathrm{~ns}$ Langevin dynamics to control the kinetic energy, temperature, and/or pressure of the system without a binding pocket, and the applying parameters do not change the protein conformation during the ligand unbinding (Feller et al. 1995; Pradeepkiran et al. 2015). MD simulation results were analyzed thoroughly for each of the FtsZ-ligand complexes and results were analyzed using VMD.

Root mean square deviation (RMSD) and root men square fluctuation (RMSF) were calculated to address the stability of atoms in the docked structures. The force field description of the interatomic forces reflects two important categories viz., the bonded terms and the non-bonded terms. The bonded terms are further regroup as simple covalent binding as well as the more complex hybridization and $\pi$-orbital effects, these are the bonds, angles, dihedrals and improper-dihedrals terms. Thus the bonding terms bonds, angles, dihedrals and improperdihedrals were plotted using NAMD plots.

\subsection{Drug likeness of the small molecules}

It is essential to predict ADME (Absorption, Distribution, Metabolism, and Excretion) properties of lead like small molecule to assess their drug likeliness. It reduces the probability of failure of a molecule as a drug candidate in the first and last stem of drug discovery (Kant et al. 2019). The selected small molecules as inhibitors of FtsZ of Mtb were analyzed for 
physicochemical and pharmacokinetics properties using the SwissADME web server (Daina et al. 2017).

\subsection{Result}

\subsection{Retrieval of rpoB and domain analysis}

The unambiguous length of the FtsZ of M. tuberculosis is 379 amino acids long (Figure 1). FtsZ is solely responsible for cell division. In the genus Mycobacterium the development of septation is driven by the cytokinetic protein FtsZ. InterProScan elucidated that protein belongs to cell division protein (IPR000158). InterProScan and CDD predicted 3 different domains in the FtsZ protein of M. tuberculosis. The domains are Tubulin_FtsZ_GTPase spanning from amino acid 10-230 (IPR003008), Tubulin/FtsZ_2-layer-sand-domain spanning from 204-321 (IPR018316) and FtsZ_C spanning from 219-313 (IPR024757).

\subsection{Docking and visualization}

Molecule 24284406

Docking between FtsZ and 24284406 showed a strong binding pattern (Figure 2a,b). The binding free energy for this docked complex was derived as -7.23. A total of 3 types of bonds were detected in FtsZ-24284406 docking complex. These bonds were Van der Waals interaction, hydrogen bonds and Pi-sigma bonds. Van der Waals interactions were observed at amino acid positions Gly-17, Asn-41, Thr-42, Asp-43, Leu-66, Gly-67, Gly-69, Gly-107 and Arg-140 (Figure 2b). There were 10 hydrogen bonds were observed in this interaction. Out of these 7 were conventional hydrogen bonds found to associate with amino acid positions Ile-16, Gly-20, Ala-68, Ala-70, Gly-101, Gly-105 and Thr-106, two carbon hydrogen bonds were observed at amino acid positions Gly-18 and Gly-19 further one Pi donor hydrogen bond was observed at amino acid position Ala-100 (Figure 2b). One Pi-sigma bond was formed at amino acid position Gly-104 (Figure 2b). The bond lengths for each bond types were given in table1. Molecule 49671233

Docking between FtsZ and 49671233 showed a strong binding pattern (Figure 3a,b). The binding free energy for this docked complex was derived as -9.67. A total of 5 types of bonds were detected in FtsZ-49671233 docking complex. These bonds were Van der waals interaction, hydrogen bonds, Pi-Anion bonds, Alkyl bonds, Pi-Alkyl bonds. Vander Waals interactions were observed at 12 amino acid positions viz., Ile-16, Gly-18, Gly-19, Gly-20, Asn-22, Asn-41, Gly-67, Gly-69, Ala-70, Gly-101, Gly-104 and Gly-105 (Figure 3b). Seven hydrogen bonds were observed at different amino acid positions of this complex. Out of 7 hydrogen bonds 5 were conventional hydrogen bonds formed at amino acid positions Thr-42, Ala-68, Thr-106, Gly-107 and Arg-140, further 2 carbon hydrogen bonds were observed at 
amino acid positions Gly-17 and Leu-66 (Figure 3b). One Pi-anion bond was observed at amino acid position Asp-43 (Figure 3b). One Alkyl bond was observed at Ala-100 and one Pi-alkyl bond was formed at amino acid position Ala-46 (Figure 3b). The bond lengths for each bond types were given in table1.

Molecule 24791139

Docking between FtsZ and 24791139 showed a strong binding pattern (Figure 4a,b). The binding free energy for this docked complex was derived as -5.51. A total of 5 types of bonds were detected in Ftsz-24791139 docking complex. These bonds were Van der waals interaction, hydrogen bonds, Florine halogen bond, Pi-anion bond and Pi-Alkyl bonds. Van der waals interactions were observed at amino acid positions Gly-17, Gly-18, Gly-19, Gly-20, Leu66, Gly-67, Ala-68, Gly-69, Ala-100, Gly-102, Gly-104, Gly-105, Gly-107 and Thr-108 (Figure $4 \mathrm{~b}$ ). The docking complex was stabilized by four conventional hydrogen bonds at amino acid positions Ala-70, Gly-101, Arg-104 and Thr-106 (Figure 4b). We observed one fluorine halogen bond at amino acid position Glu-136 (Figure 4b). One Pi-anion bond was observed at amino acid position Glu-136 and two Pi-alkyl bonds were observed at amino acid positions Ala-70 and Phe-180 (Figure 4b). The bond lengths for each bond types were given in table1.

\subsection{Molecular dynamics (MD) simulation of FtsZ and small molecule complex}

Molecular Dynamics simulation is a computer based theoretical technique which is used to understand the physical movements of atoms and molecules. The technique is frequently used for assessing and evaluating the stability of a predicted model in protein science. In this study MD simulation was used to understand the affinity of ligands towards FtsZ protein and stability of docking conformations.

The RMSD of protein-ligand backbone of docked conformations were $<1.45 \AA$ for each docking complex in 100 ns MD runs (Figure 5). The average RMSD of FtsZ-24284406 complex was $1.011 \AA$ with minimum of $0.073 \AA$ and maximum of $1.348 \AA$. The average RMSD of FtsZ-49671233 complex was $0.978 \AA$ with minimum of $0.069 \AA$ and maximum of $1.195 \AA$ and the average RMSD of FtsZ-24791139 complex was $1.136 \AA$ with minimum of $0.077 \AA$ and maximum of $1.440 \AA$ (Table 2). The backbones of protein-ligand structure were found to be stable in all the three MD trajectories. The average RMSF of residues contributing to the complex structure in each of the docked conformations were calculated as > $1.43 \AA$ (Figure 6). The RMSF of each docked conformations for FtsZ-24284406, FtsZ-49671233 and FtsZ24791139 were $<1.43 \AA$, $<1.39 \AA$ and $<1.38 \AA$ respectively (Table 2). Slight fluctuations were observed at amino acid positions Val-54, Asp-57, Gly-59, Ser-62, and Ile-290 for FtsZ- 
24284406 complex; Met-27, Val-54, Lys-55, Asp-57, Leu-166, Val-279 and Arg-304 for FtsZ49671233; Asn-163, Asp-164, Arg-165, and Gly-292 for FtsZ-24791139 complex. The bonded and non bonded terms like bonds, angles, dihedrals and improper-dihedrals, kinetic energy, temperature and average pressure showed that the FtsZ-ligand docked structures are stable in each of the parameters tested.

\subsection{Principal Component Analysis}

Principal component analysis (PCA) helps in recognition of binding affinity of biomolecular systems by using local and global motion of protein component. Highly correlated fluctuations are extracted from MD trajectory using dimensional reduction (Chang et al 2010). In FtsZ24284406 complex the first three components were responsible for $47.31 \%$ of the total variance (Figure 7a). In FtsZ-49671233 complex the first three components were responsible for $47.33 \%$ of the total variance (Figure 7b) while in FtsZ-24791139 complex the first three components were responsible for $50.54 \%$ of the total variance (Figure 7c). The percentages of variation for each of the docking complex are shown in table 2. Visualization of PC1 using VMD showed slow motion in C-terminus for each docking complex.

\subsection{Drug likeness of the small molecules}

SWISS-ADME was used to assess the drug likeness and pharmacokinetics properties of the molecules. In the SWISS-ADME analysis all the three molecules showed reasonable amount of drug likeliness and pharmacokinetics properties. Slight deviation in molecular weight was observed in case of compound 49671233 and 24791139. The results were shown in table 3.

\subsection{Discussion}

TB is a major health problem in developing and lower income countries worldwide. TB is one of the top 10 causes of death worldwide and the leading cause of death from a single infectious agent (WHO 2020). Nearly one-fourth of the world population is infected with TB. WHO set the goal to end TB by 2035 in a phased manner. In the first phase the target was set to reduce $35 \%$ of total TB cases by $2020,75 \%$ reduction by 2025 in the second phase, $90 \%$ reduction by 2030 in the third phase, and 95\% reduction by 2035 in the fourth phase (WHO, 2018). Occurrence of drug resistant TB including MDR and XDR-TB are a major concern for the end TB strategy. Drug-resistant TB continues to be a public health threat and put obstacles in achieving eradication of the disease worldwide. In 2019 nearly half a million (465,000 in absolute number) people were infested with rifampicin resistant TB (RR-TB), of which 78\% $(362,700)$ had multidrug resistant TB (MDR-TB). In 2019, 3.3\% of total new TB cases and $17.7 \%$ of previously treated cases had MDR/RR-TB (WHO, 2020). An effecting control of infectious disease needs either a good vaccine or regimens those can control the disease 
effectively. A number of new TB vaccines including genetically modified mycobacteria, mycobacterial antigens with or without adjuvant are in clinical trials phase. BCG is the only vaccine approved for TB and given just after the birth. According to some study BCG vaccine provides incomplete and variable protection against pulmonary TB (Dockrell and Smith 2017; Barreto et al. 2006; Arbelaez et al. 2000, Van-Dunem et al. 2015). Thus looking to the magnitude of the TB problem worldwide discovery of new cost effective, direct effect and having less side effect drugs is an important target for TB elimination programme. Thus in the present study an attempt has been made to screen lead like small molecules against FtsZ, a cell cycle regulation protein Mtb using structure based drug screening.

In the present study, a 3D model of FtsZ protein of Mtb was downloaded from protein databank and had been used for virtual screening of potential inhibitors from diverse-lib implemented in MTiOpenScreen server (Labbe et al. 2018).

From the molecular interaction study, it was observed that the selected drug molecules viz., 24284406, 49671233 and 24791139 had strong affinities towards the FtsZ. This was evident from the free binding energy, bonding pattern and types of bonds involved in ligand and FtsZ interaction. The molecular interaction study showed a strong binding association exists between FtsZ and interacting drug molecules through hydrogen bonds, van der Waals interaction, Pi-Sigma bonds, alkyl bonds, Pi-alkyl interactions, halogen bond and Pi-lone pair interactions. Hydrogen bonds are major player for negative free energy change and contribute to the stability of protein-ligand binding interface complex (Fu et al. 2018).

Hydrogen bonds are also known to enhance the protein ligand interaction at the binding interface (Chen et al. 2016). In this study we observed 7 conventional hydrogen bonds and 2 carbon hydrogen bonds for FtsZ-24284406; 5 conventional hydrogen bonds and 2 carbon hydrogen bonds for FtsZ-49671233 and four conventional hydrogen bonds for FtsZ-24791139 docking complexes. These interactions showed a strong association and stabilization of FtsZligand interface.

Though van der Waals interactions are weaker than covalent and electrostatic bonding but this bond in large is responsible for free energy change and made the major contribution to the total binding energies (Sood et al. 2018; Mohanty et al. 2018; Keretsu et al. 2019). These interactions are known to stabilize the binding interface structures through intermolecular energy minimization (Kumar et al. 2015; Arthur and Uzairu 2019). In this study we observed a large number of van der Waals interactions stabilizing the FtsZ and interacted small molecule docking complex. We observed 9, 12 and 14 van der Waals interactions between FtsZ and 24284406,49671233 and 24791139 docked structures respectively. Thus we summarised that 
these van der Waals interactions were largely contributed to the negative free energy change and stabilize the FtsZ and each lead like molecule docked interface.

Other key interactions those are responsible for negative free energy change and provide stability to protein-ligand interface are alkyl bonds, Pi-alkyl interactions, Pi-Sigma bonds, and Pi-lone pair interactions. Pi-alkyl bonds which involve in charge transfer play a key role in intercalating the drugs in the binding site of the receptor ${ }^{33}$. These essential Pi-alkyl bonds were observed in this study. Further the conformational stability of the protein-ligand interactions are derived from Pi-lone, Pi-anion and halogen non covalent interactions (Brylinski 2018; Arthur and Uzairu 2019; Belhassan et al. 2019). In this study we observed Pi-sigma interaction in the interface of FtsZ-24284406; Pi-anion bond was observed in FtsZ-49671233 and FtsZ24791139 complex and one fluorine bond was observed in FtsZ-49671233 complex. From the molecular docking study it was evident that 3 selected lead molecules viz., 24284406, 49671233 and 24791139 would be able to inhibit the replication of Mtb.

The RMSD trajectory is used to predict the stability of the protein-ligand complex. Lower the RMSD value indicates high stability of the protein-ligand complex structure (Kumar et al., 2014). The RMSD values for all 3 FtsZ-ligand complexes were less than $1.45 \AA$ and showing the stable conformations of the docked structures. The RMSF of FtsZ-ligand complexes were calculated to determine the flexibility of the docked backbone after docking (Kumar et al. 2014). In this study higher RMSF value (> $1 \AA$ ) showed the more flexibility movement of residues in the docked backbone. This indicates the higher level conformation structures of FtsZ-ligand complexes. The stability of the docking conformations are also implied from the stable values of bonded parameters like bonds, angles, dihedrals and improper-dihedrals, kinetic energy, temperature and average pressure. Principal component analysis (PCA) is a better tool to determine the relationship between statistically meaningful conformations or major global motions sampled during the trajectory formation in a docked complex (McCammon and Harvey 1986). From the PCA analysis it was found that the global motions and conformational changes in the protein backbone were least for all the three docked structures. On the basis of least motion rank wise best conformations were FtsZ-24284406, FtsZ-49671233 and FtsZ-24791139 respectively. These findings were also supported by the cosine content value of each PCA coordinates, i.e., less than 6 for all three docking complexes (Maisuradze et al. 2007).

It is an essential to predict absorption, distribution, metabolism, excretion and toxicity of any lead like molecule to understand the action, behaviour and bioavailability of the molecules before entering into a clinical experimentation. In this study SWISS-ADME was employed to 
all the proposed molecules to assess the toxicity and bioavailability of the lead like molecules. We found that all the three molecules passed the ADME test could be used for clinical experimentation against M.tb.

The molecular interaction study showed that a strong binding association exists between modelled protein FtsZ and interacting lead molecules. The molecular interaction patterns between the drug molecules and one of the binding site residues (Gly-18, Gly-19, Asn-22, Gly105 and Thr-106) of FtsZ might interrupt protein function.

\section{Acknowledgement}

We are highly thankful to Indian Council of Medical Research for providing financial and logistic support for the study.

\section{References}

AlMatar M, AlMandeal H, Var I, Kayar B, Köksal F (2017) New drugs for the treatment of Mycobacterium tuberculosis infection. Biomed Pharmacother 91:546-558.

Anderson LF, Tamne S, Brown T, Watson JP, Mullarkey C, Zenner D, Abubakar I (2014) Transmission of multidrug-resistant tuberculosis in the UK: a cross-sectional molecular and epidemiological study of clustering and contact tracing. Lancet Infect Dis 14:406-415.

Arbeláez MP, Nelson KE, Muñoz A (2000) BCG vaccine effectiveness in preventing tuberculosis and its interaction with human immunodeficiency virus infection. Int J Epidemiol 29:1085-1091.

Arthur DE, Uzairu A (2019) Molecular docking studies on the interaction of NCI anticancer analogues with human Phosphatidylinositol 4, 5-bisphosphate 3-kinase catalytic subunit. J King Saudi Univ Sci 31:1151-1166.

Barreto ML, Pereira SM, Ferreira AA (2006) BCG vaccine: efficacy and indications for vaccination and revaccination. J Pediatr 82:45-54.

Belhassan A, Zaki H, Benlyas M, Lakhlifi T, Bouachrine M (2019) Study of novel triazolobenzodiazepine analogues as antidepressants targeting by molecular docking and ADMET properties prediction. Heliyon 5:e02446.

Bi E, Lutkenhaus J (1991) FtsZ ring structure associated with division in Escherichia coli. Nature 354:161-164.

Brylinski M (2018) Aromatic interactions at the ligand-protein interface: Implications for the development of docking scoring functions. Chem Biol Drug Design 91:380-390.

Chen D, Oezguen N, Urvil P, Ferguson C, Dann SM, Savidge TC (2016) Regulation of proteinligand binding affinity by hydrogen bond pairing. Sci Adv 2:e1501240. 
Daina A, Michielin O, Zoete V (2017) SwissADME: a free web tool to evaluate pharmacokinetics, drug-likeness and medicinal chemistry friendliness of small molecules. Sci Rep 7:42717.

DeJesus MA, Gerrick ER, Xu W, Park SW, Long JE, Boutte CC, et al (2017) Comprehensive essentiality analysis of the Mycobacterium tuberculosis genome via saturating transposon mutagenesis. MBio 8:e02133-16.

DeLano WL (2002) Pymol: An open-source molecular graphics tool. CCP4 Newsletter on protein crystallography 40:82-92.

Dockrell HM, Smith SG (2017) What have we learnt about BCG vaccination in the last 20 years? Front Immunol 8:1134.

Feller SE, Zhang Y, Pastor RW, Brooks BR (1995) Constant pressure molecular dynamics simulation: the Langevin piston method. J Chem Phys 103:4613-4621.

Finn RD, Bateman A, Clements J, Coggill P, Eberhardt RY, Eddy SR, et al (2014) Pfam: the protein families database. Nucleic Acids Res 42:211-222.

Fox GJ, Dobler CC, Marais BJ, Denholm JT (2017) Preventive therapy for latent tuberculosis infection - the promise and the challenges. Int J Infect Dis 56:68-76.

Fu Y, Zhao J, Chen Z (2018) Insights into the molecular mechanisms of protein-ligand interactions by molecular docking and molecular dynamics simulation: a case of oligopeptide binding protein. Comput Math Method Med. 2018:3502514.

Griffin JE, Gawronski JD, DeJesus MA, Ioerger TR, Akerley BJ, Sassetti CM (2011) Highresolution phenotypic profiling defines genes essential for mycobacterial growth and cholesterol catabolism. PLoS Pathog 7:e1002251.

Gupta P, Pradhan A, Ajitkumar P (2017) Unique Biochemical Features of the Cytokinetic Protein FtsZ of Mycobacteria. In Mycobacterium-Research and Development. Dec 20. IntechOpen.

Huang Q, Tonge PJ, Slayden RA, Kirikae T, Ojima I (2007) FtsZ: a novel target for tuberculosis drug discovery. Curr Top Med Chem 7:527-543.

Humphrey W, Dalke A, Schulten K (1996) VMD: visual molecular dynamics. J Mol Graph 14:33-38.

Jo S, Kim T, Iyer VG, Im W (2008) CHARMM-GUI: a web-based graphical user interface for CHARMM. J Comput Chem 29:1859-1865.

Kant K, Lal UR, Kumar A, Ghosh M (2019) A merged molecular docking, ADME-T and dynamics approaches towards the genus of Arisaema as herpes simplex virus type 1 and type 2 inhibitors. Comput Biol Chem 78:217-226. 
Keretsu S, Bhujbal SP, Cho SJ (2019) Computational study of paroxetine-like inhibitors reveals new molecular insight to inhibit GRK2 with selectivity over ROCK1. Sci Rep 9:13053.

Kumar CV, Swetha RG, Anbarasu A, Ramaiah S (2014) Computational analysis reveals the association of threonine 118 methionine mutation in PMP22 resulting in CMT-1A. Adv Bioinform 2014:502618.

Kumar P, Choonara YE, Pillay V (2015) In silico affinity profiling of neuroactive polyphenols for post-traumatic calpain inactivation: a molecular docking and atomistic simulation sensitivity analysis. Molecules 20:135-168.

Labbé CM, Rey J, Lagorce D, Vavruša M, Becot J, Sperandio O, et al (2015) MTiOpenScreen: a web server for structure-based virtual screening. Nucleic Acids Res 43:W448-454.

Leung AK, White EL, Ross LJ, Reynolds RC, DeVito JA, Borhani DW (2004) Structure of Mycobacterium tuberculosis FtsZ reveals unexpected, G protein-like conformational switches. J Mol Biol 342:953-970.

Li Z, Trimble MJ, Brun YV, Jensen GJ (2007) The structure of FtsZ filaments in vivo suggests a force-generating role in cell division. EMBO J 26:4694-708.

Maisuradze GG, Leitner DM (2007) Free energy landscape of a biomolecule in dihedral principal component space: sampling convergence and correspondence between structures and minima. Proteins Struct Funct Bioinf 67:569-578.

Marchler-Bauer A, Anderson JB, Chitsaz F, Derbyshire MK, Deweese-Scott C, Fong JH, et al (2009) CDD: specific functional annotation with the Conserved Domain Database. Nucleic Acids Res 37:205-210.

McCammon AJ, Harvey SC (1986) Dynamics of Proteins and Nucleic Acids, Cambridge University Press, Cambridge.

Mohanty PS, Bansal AK, Naaz F, Gupta UD, Dwivedi VD, Yadava U (2018) Ribonucleotide reductase as a drug target against drug resistance Mycobacterium leprae: A molecular docking study. Infect Genet Evol 60:58-65.

Morris GM, Huey R, Lindstrom W, Sanner MF, Belew RK, Goodsell DS, Olson AJ (2009) AutoDock4 and AutoDockTools4: Automated docking with selective receptor flexibility. J Comput Chem 30:2785-2791.

Nogales E, Whittaker M, Milligan RA, Downing KH (1999) High-resolution model of the microtubule. Cell 96:79-88.

Pradeepkiran JA, Kumar KK, Kumar YN, Bhaskar M (2015) Modeling, molecular dynamics, and docking assessment of transcription factor rho: a potential drug target in Brucella melitensis 16M. Drug Des Develop Ther 9:1897-1912. 
Seung KJ, Keshavjee S, Rich ML (2015) Multidrug-resistant tuberculosis and extensively drug-resistant tuberculosis. Cold Spring Harb Perspect Med 5:a017863.

Sood D, Kumar N, Rathee G, Singh A, Tomar V, Chandra R (2018) Mechanistic interaction study of bromo-noscapine with bovine serum albumin employing spectroscopic and chemoinformatics approaches. Sci Rep 8:16964.

Trott O, Olson AJ (2010) AutoDock Vina: improving the speed and accuracy of docking with a new scoring function, efficient optimization, and multithreading. J Comput Chem 31:455461.

Van-Dunem JC, Rodrigues LC, Alencar LC, Militão-Albuquerque MD, Ximenes RA (2015) Effectiveness of the first dose of BCG against tuberculosis among HIV-infected, predominantly immunodeficient children. BioMed Res Int 29: 2015.

WHO, 2018. Global tuberculosis report. World Heal. Organ, pp. 1-231.

WHO, 2020. Global tuberculosis report. World Heal. Organ, pp. 1-232.

Zdobnov EM, Apweiler R (2001) InterProScan - an integration platform for the signature recognition methods in InterPro. Bioinformatics 17:847-848.

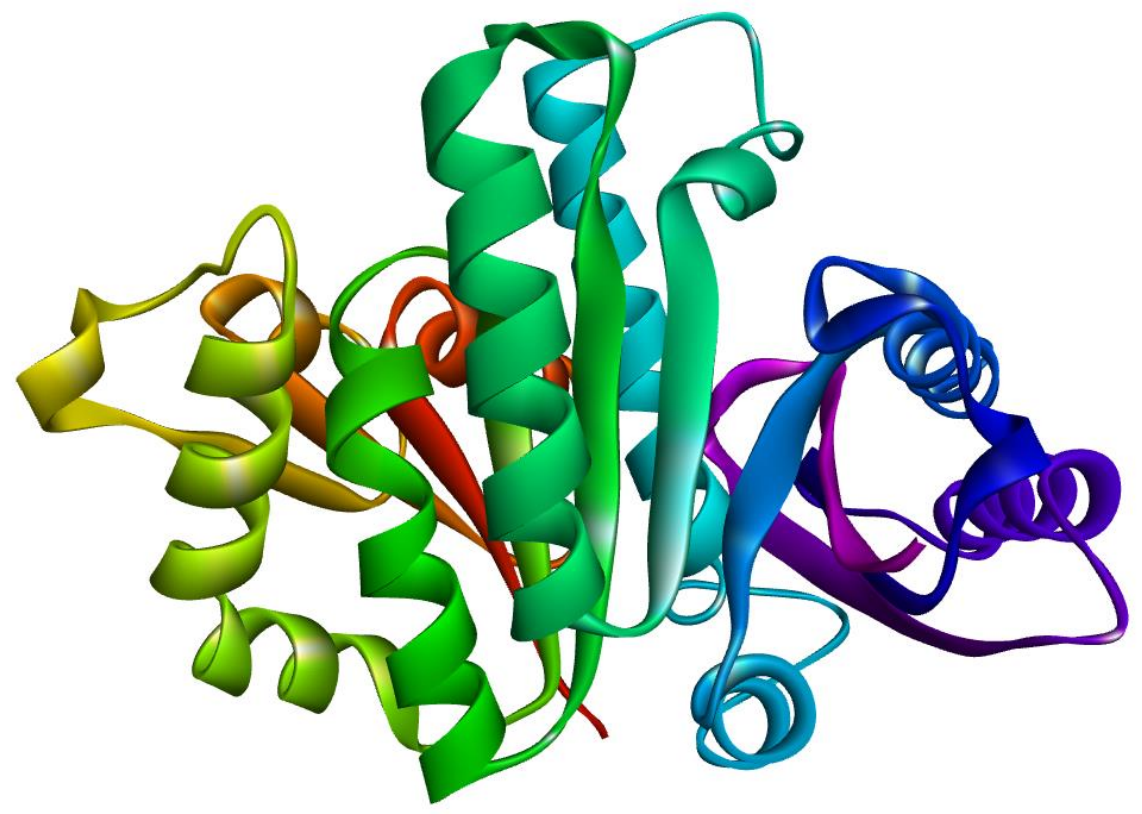

Figure 1: 3D structure of cell cycle regulator enzyme FtsZ-chain A. 

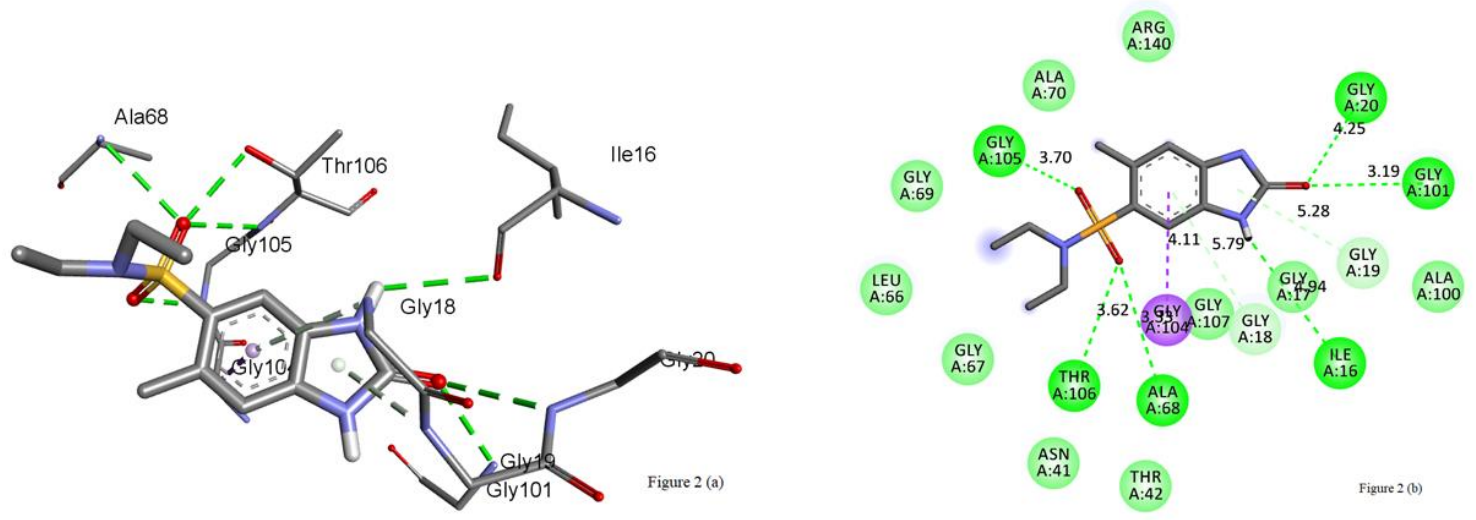

Figure 2a,b: (a) 3D representation of interaction of surface accessibility area of FtsZ and Lig24284406 (b) 2D schematic interaction diagram of FtsZ and Lig-24284406 complex showing different types of interactions along with their bond length.
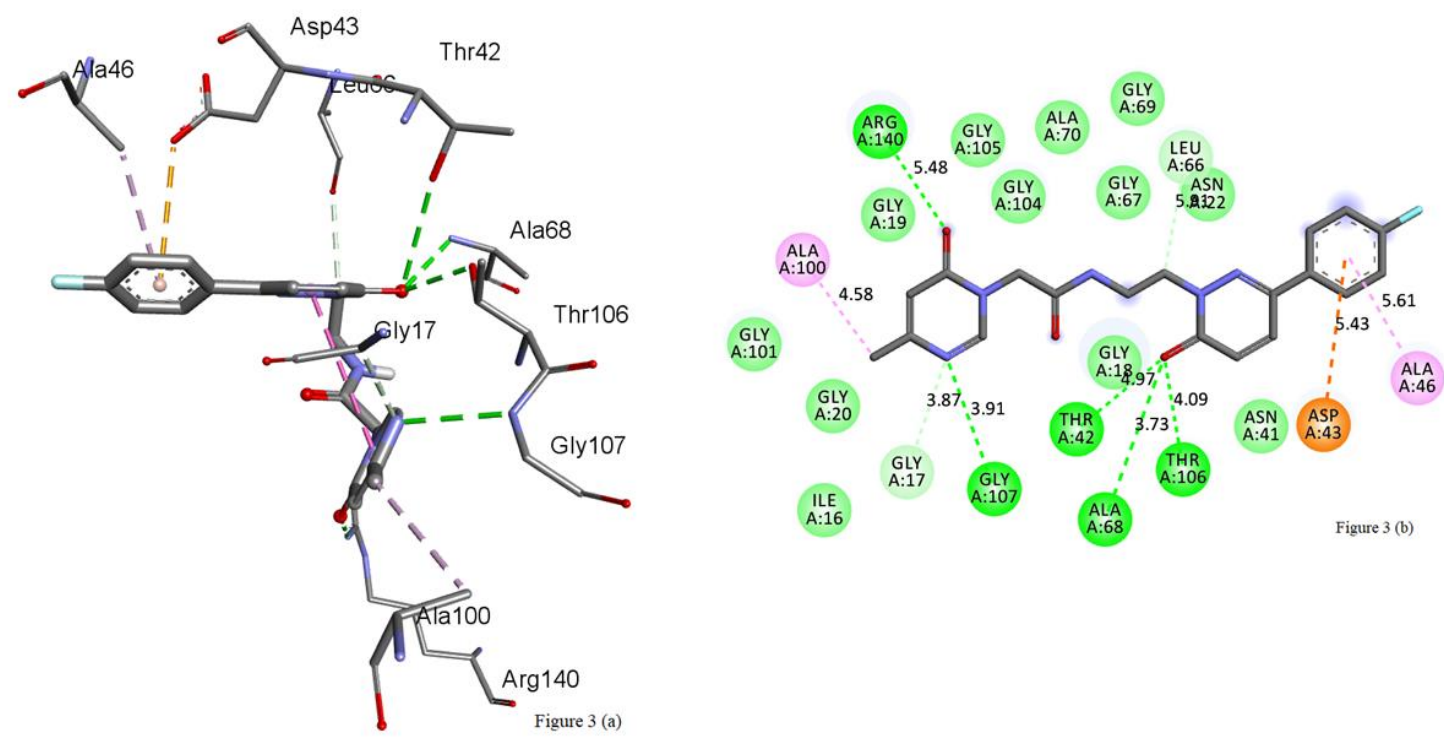

Figure 3a,b: (a) 3D representation of interaction of surface accessibility area of FtsZ and Lig49671233 (b) 2D schematic interaction diagram of FtsZ and Lig-49671233 complex showing different types of interactions along with their bond length. 

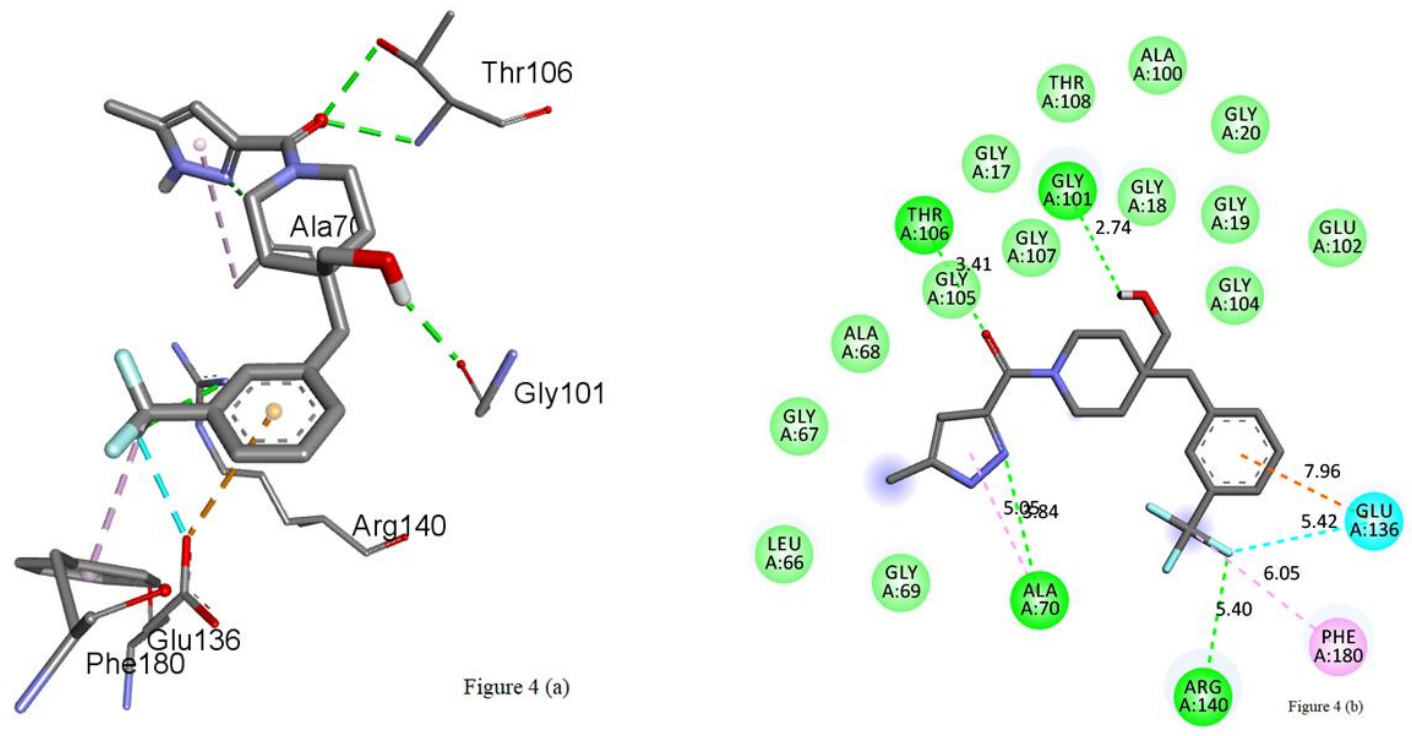

Figure 4a,b: (a) 3D representation of interaction of surface accessibility area of FtsZ and Lig24791139 (b) 2D schematic interaction diagram of FtsZ and Lig-24791139 complex showing different types of interactions along with their bond length.

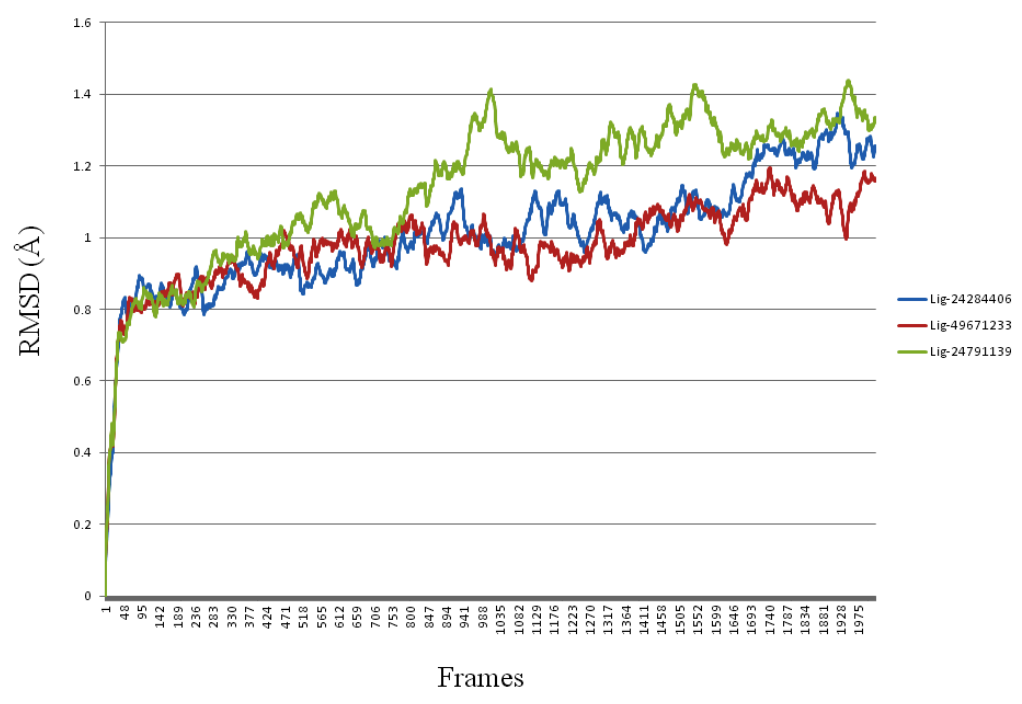

Figure 5: RMSD plot of protein backbone complexed with Lig-24284406 (Blue), Lig49671233 (Red) and Lig-24791139 (Green). 


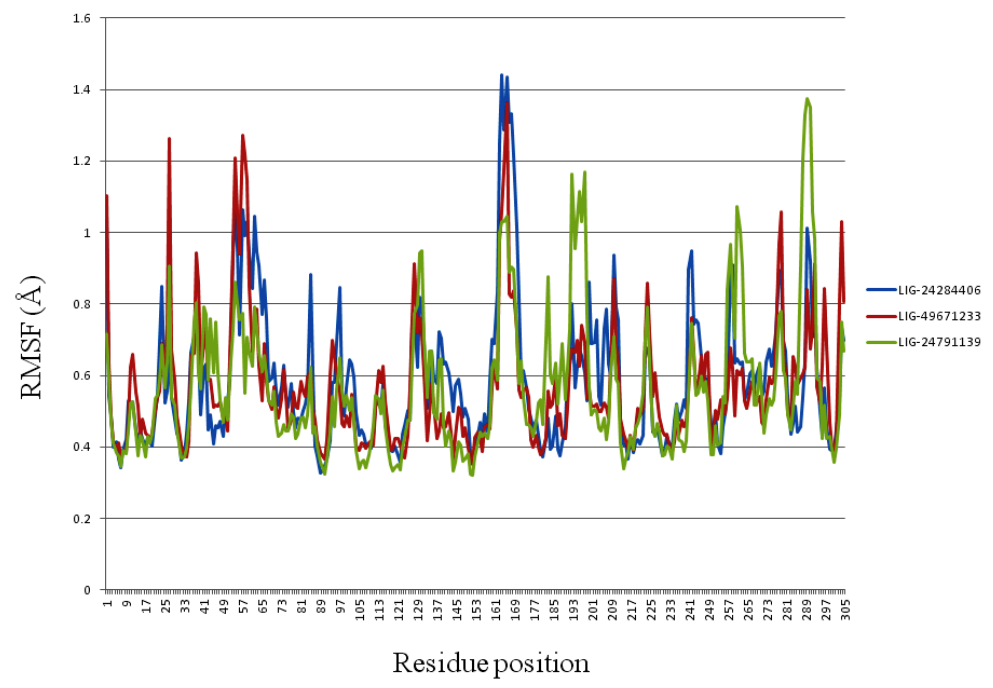

Figure 6: RMSF plot of protein backbone complexed with Lig-24284406 (Blue), Lig49671233 (Red) and Lig-24791139 (Green)
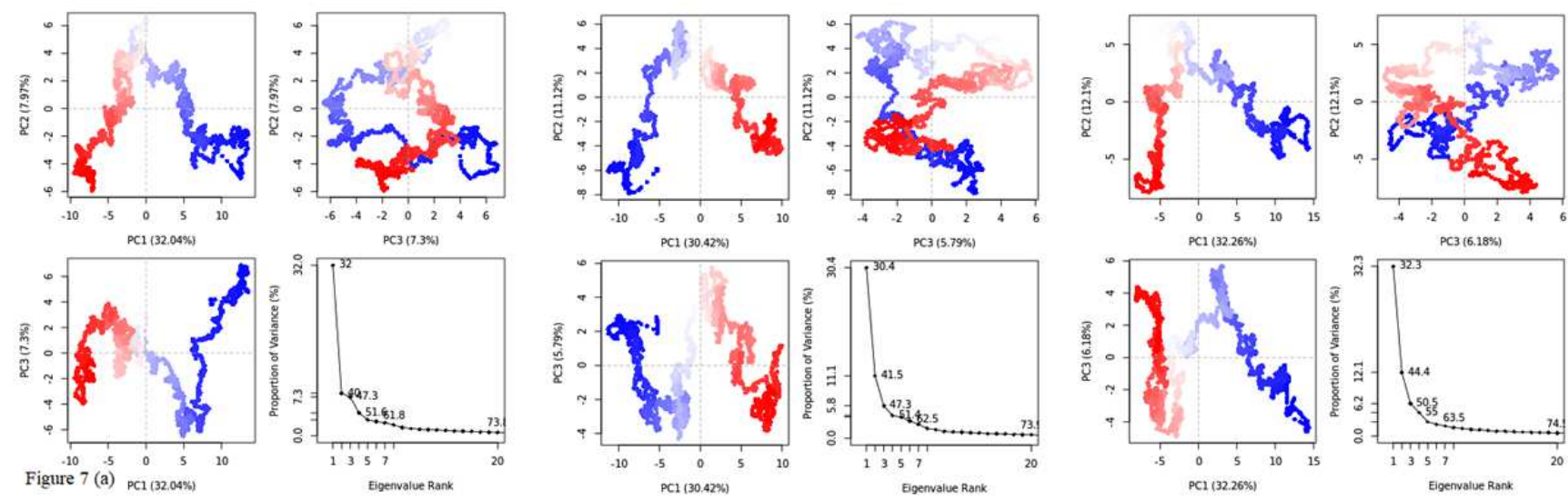

Figure 7: Principle component analysis cluster plot showing the Eigenvalue of PC1, PC2 and PC3 (a) Ftsz and Lig-24284406 complex, (b) FtsZ and Lig-24284406 complex, (c) FtsZ and Lig-24791139 complex. 


\section{Figures}

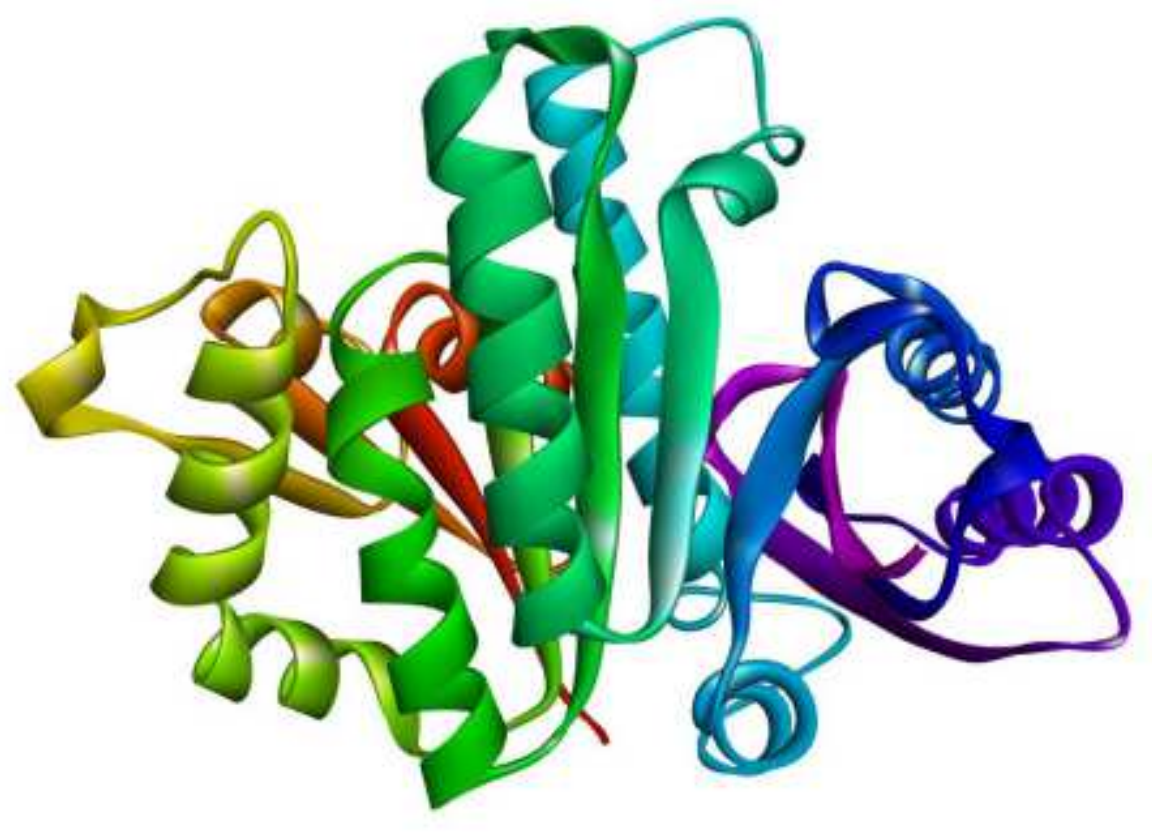

Figure 1

3D structure of cell cycle regulator enzyme FtsZ-chain A.
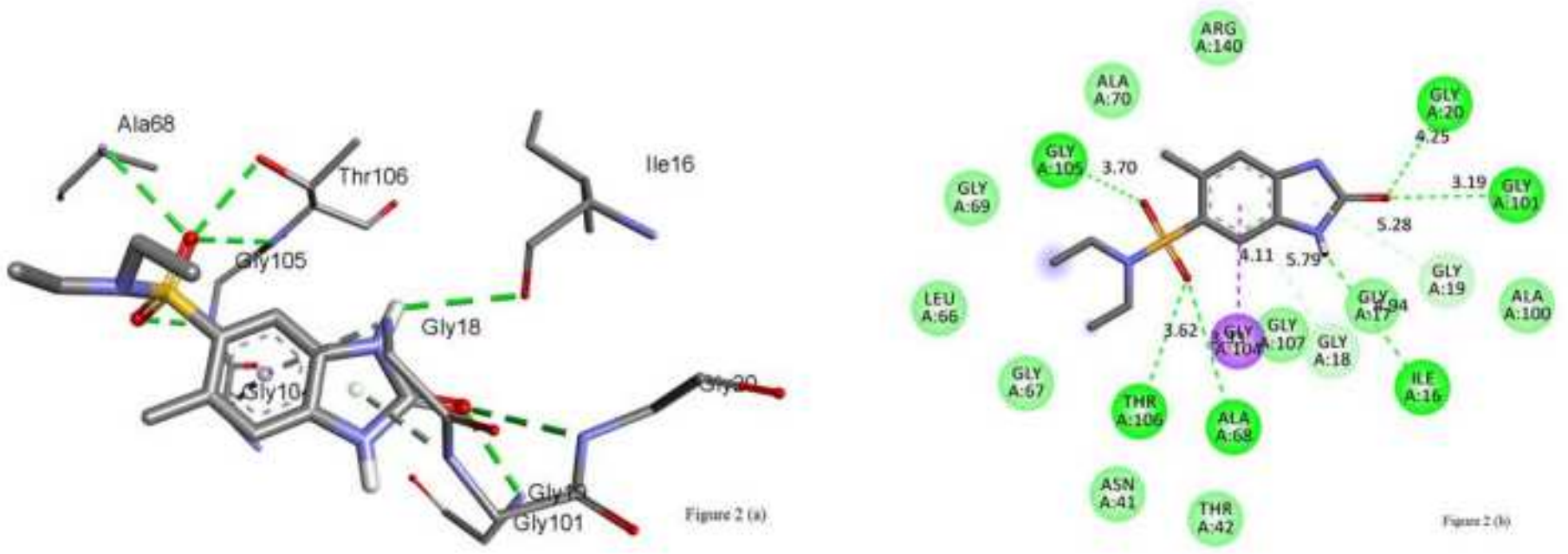

Figure 2

(a) 3D representation of interaction of surface accessibility area of FtsZ and Lig24284406 (b) 2D schematic interaction diagram of FtsZ and Lig-24284406 complex showing different types of interactions along with their bond length. 


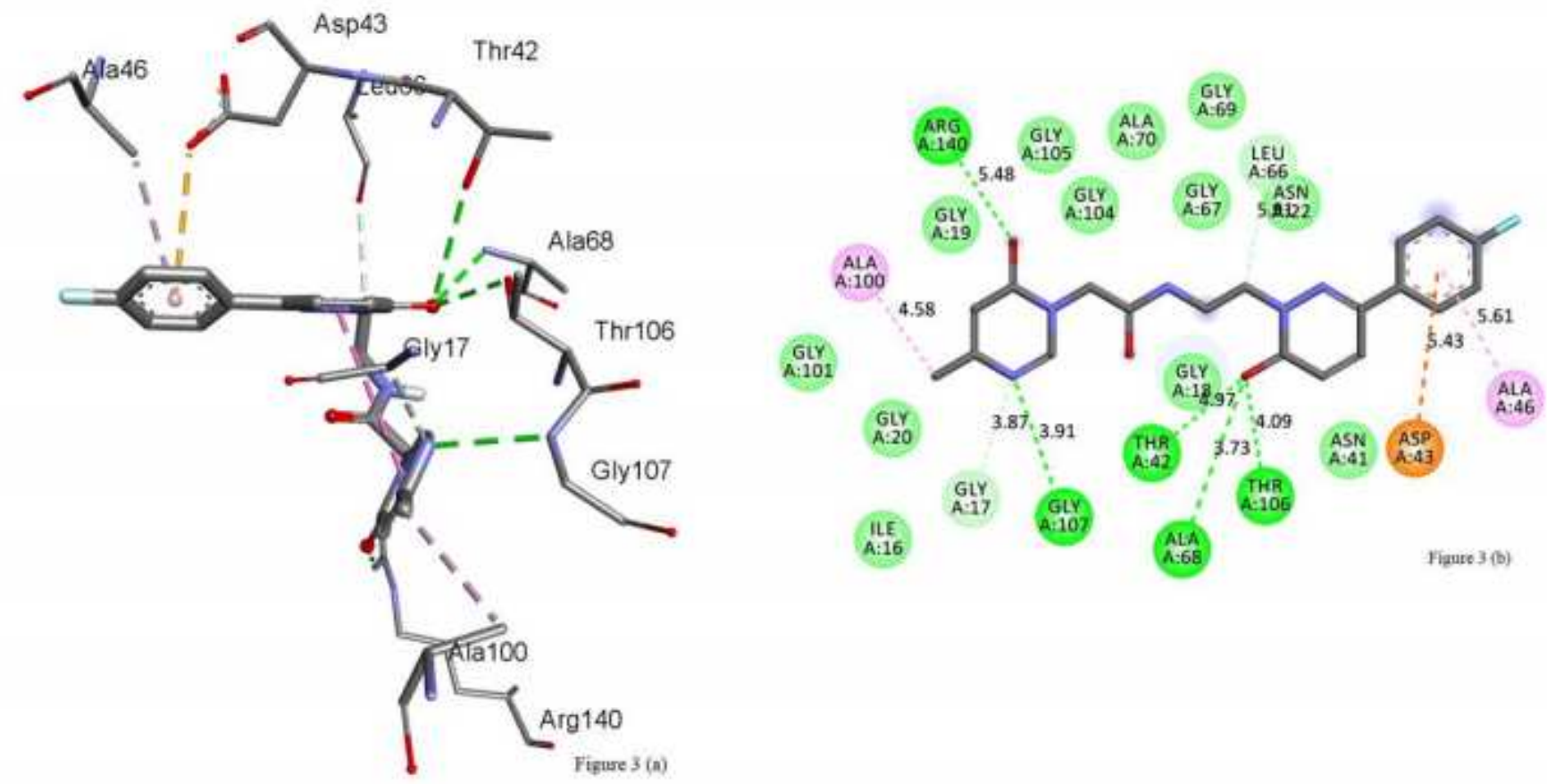

Figure 3

(a) 3D representation of interaction of surface accessibility area of FtsZ and Lig49671233 (b) 2D schematic interaction diagram of FtsZ and Lig-49671233 complex showing different types of interactions along with their bond length.
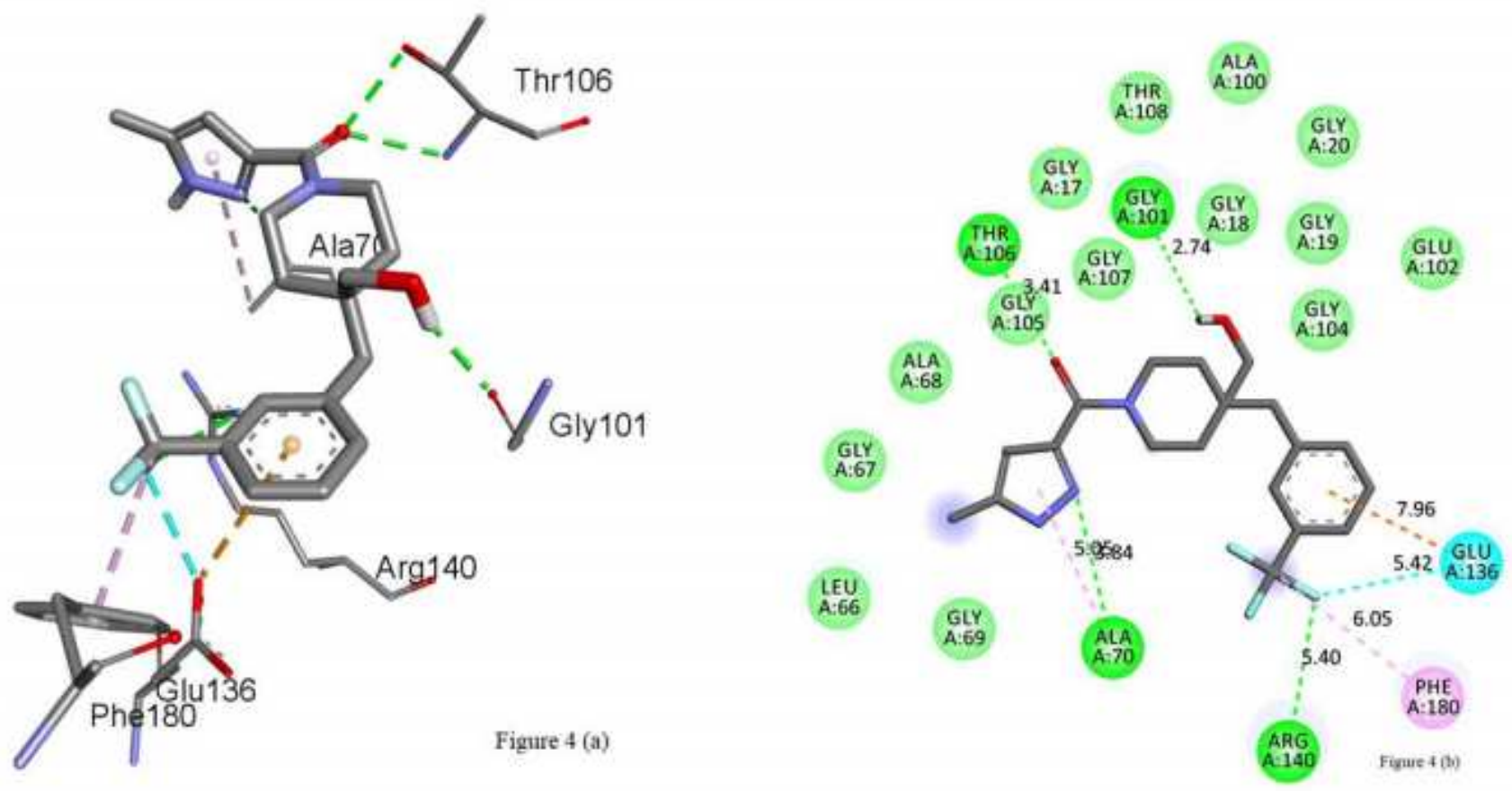

Figure 4 
(a) 3D representation of interaction of surface accessibility area of FtsZ and Lig24791139 (b) 2D schematic interaction diagram of FtsZ and Lig-24791139 complex showing different types of interactions along with their bond length.

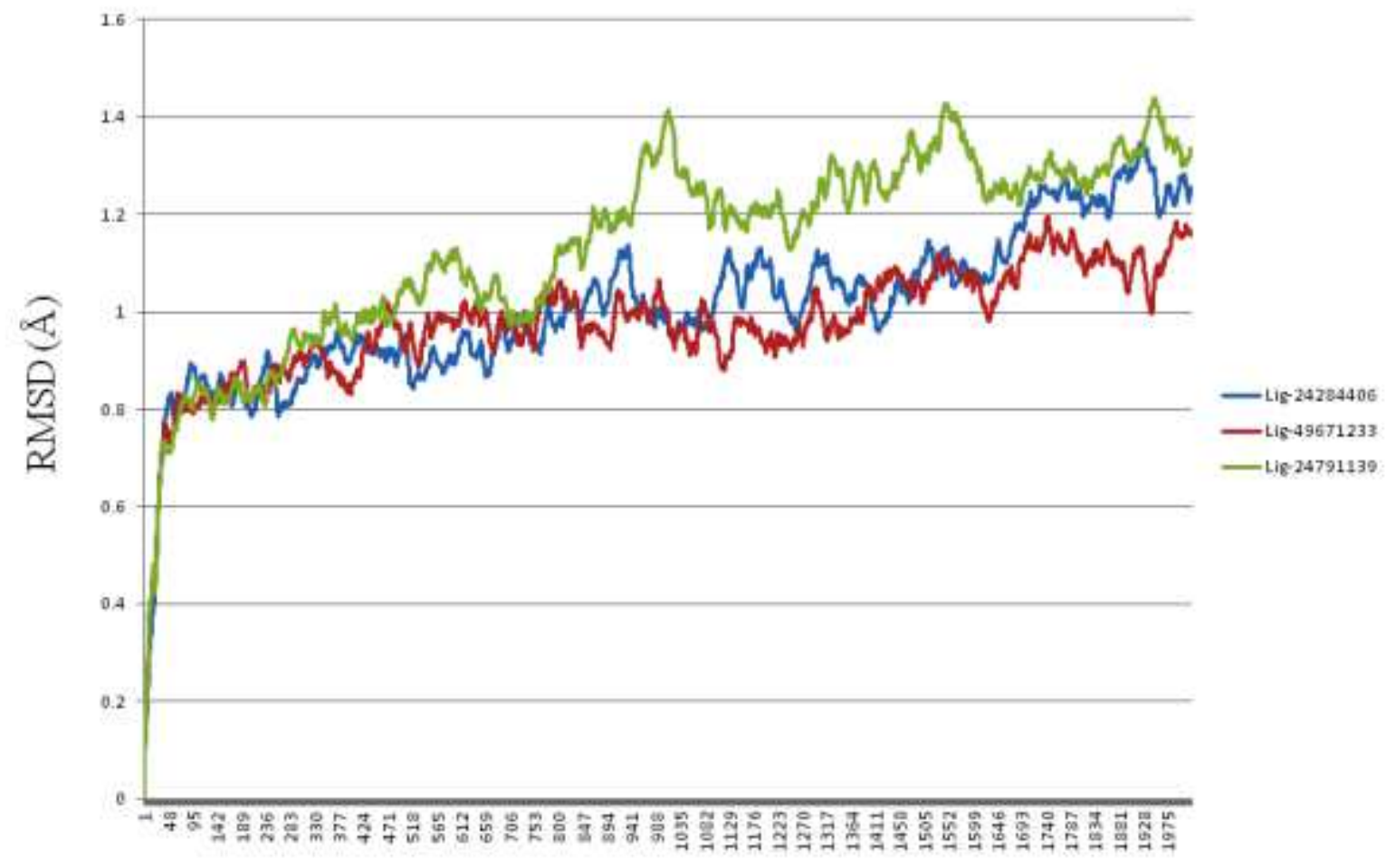

Frames

\section{Figure 5}

RMSD plot of protein backbone complexed with Lig-24284406 (Blue), Lig49671233 (Red) and Lig24791139 (Green). 


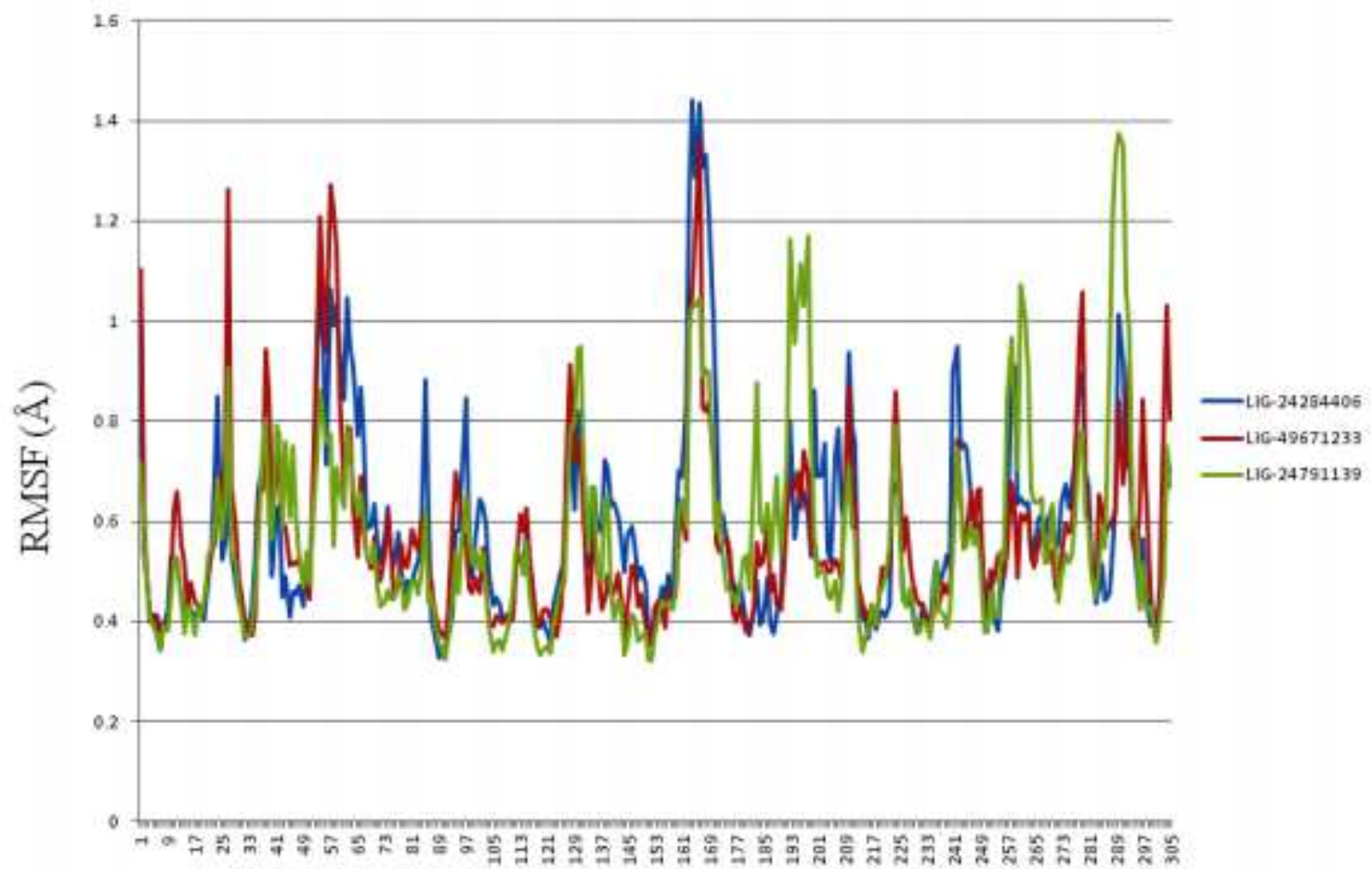

Residue position

Figure 6

RMSF plot of protein backbone complexed with Lig-24284406 (Blue), Lig49671233 (Red) and Lig24791139 (Green)
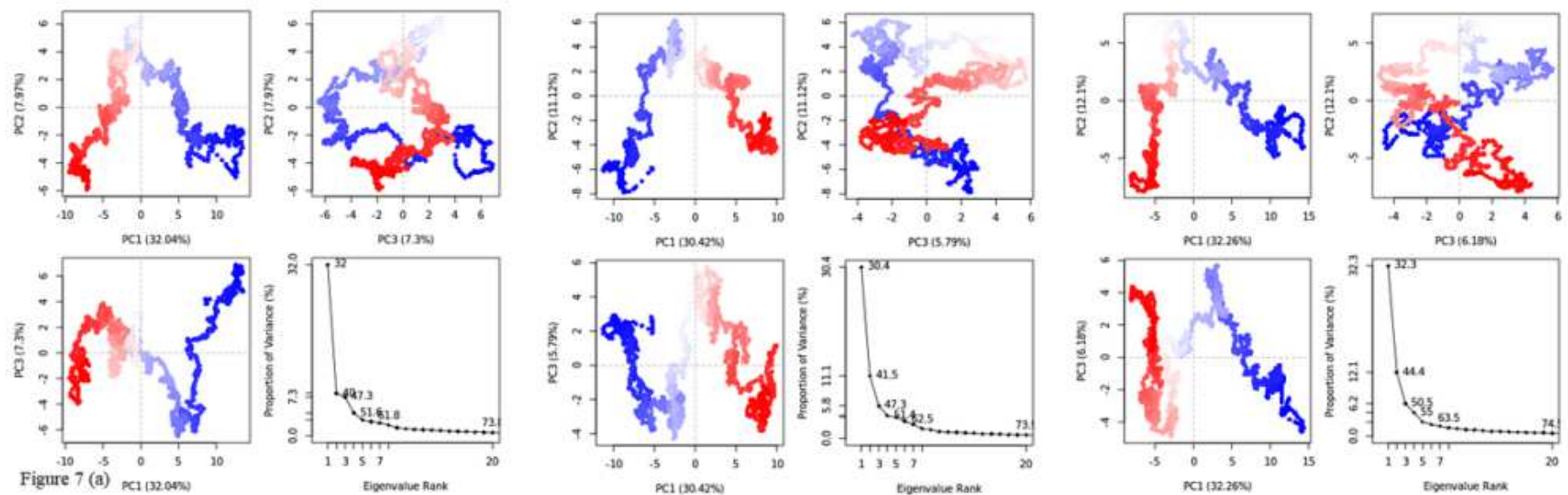

\section{Figure 7}

Principle component analysis cluster plot showing the Eigenvalue of PC1, PC2 and PC3 (a) Ftsz and Lig24284406 complex, (b) FtsZ and Lig-24284406 complex, (c) FtsZ and Lig-24791139 complex. 